\title{
Adherence to Cardiovascular Medications: Lessons Learned and Future Directions
}

\author{
Ian M Kronish, MD, MPH and Siqin Ye, MD
}

\begin{abstract}
Approximately $50 \%$ of patients with cardiovascular disease and/or its major risk factors have poor adherence to their prescribed medications. Finding novel methods to help patients improve their adherence to existing evidence-based cardiovascular drug therapies has enormous potential to improve health outcomes while potentially reducing health care costs. The goal of this report is to provide a review of the current understanding of adherence to cardiovascular medications from the point of view of prescribing clinicians and cardiovascular researchers. Key topics addressed include: 1) definitions of medication adherence; 2) prevalence and impact of non-adherence; 3) methods for assessing medication adherence; 4) reasons for poor adherence; and 5) approaches to improving adherence to cardiovascular medications. For each of these topics, the report seeks to identify important gaps in knowledge and opportunities for advancing the field of cardiovascular adherence research.
\end{abstract}

\section{Keywords}

medication adherence; cardiovascular disease

\section{Introduction}

Modern medicine has been notable for its discovery of numerous medications that improve health outcomes. For example, since the 1950s, nearly one hundred blood pressure medications from twelve distinct classes have been developed. Yet, despite the clear benefits of antihypertensive medications on blood pressure control and cardiovascular outcomes, up to $50 \%$ of patients do not take their blood pressure medications as prescribed and only $50 \%$ of treated individuals reach their blood pressure goals. ${ }^{1}$ Adherence to other cardiovascular medications such as statins and antiplatelets is equally poor. ${ }^{1,2}$ Compared with all of the resources spent on the development of new drugs, helping patients improve their adherence to existent cardiovascular drugs has enormous potential for improving health outcomes while reducing healthcare costs.

The goal of this report is to provide a review of the current understanding of adherence to cardiovascular medications from the point of view of prescribing clinicians and cardiovascular researchers. Key topics addressed include: 1) definitions of medication

(C) 2013 Elsevier Inc. All rights reserved.

Correspondence: Ian M Kronish, MD, MPH, Assistant Professor of Medicine, Center for Behavioral Cardiovascular Health, Columbia University Medical Center, 622 West $168^{\text {th }}$ Street, PH9-311, New York, NY 10032, Phone: 212-342-1335; Fax 212-305-0312; ik2293@columbia.edu.

Publisher's Disclaimer: This is a PDF file of an unedited manuscript that has been accepted for publication. As a service to our customers we are providing this early version of the manuscript. The manuscript will undergo copyediting, typesetting, and review of the resulting proof before it is published in its final citable form. Please note that during the production process errors may be discovered which could affect the content, and all legal disclaimers that apply to the journal pertain. 
adherence; 2) prevalence and impact of non-adherence; 3) methods for assessing adherence; 4) reasons for poor adherence; and 5) approaches to improving adherence to cardiovascular medications. For each of these topics, the report seeks to identify important gaps in knowledge and opportunities for advancing the field of cardiovascular adherence research.

\section{Defining Medication Adherence}

Medication adherence refers to the extent to which patients take medications in the manner prescribed by their clinicians. ${ }^{3}$ For the most part, the term "adherence" has replaced the term "compliance" in the medical literature. This change in terminology reflects the contemporary, patient-centered concept of health care in which patients and providers collaborate on treatment plans, rather than patients unilaterally following physicians' instructions. There are more precise definitions of adherence that pertain to different aspects of medication adherence. The first pertains to the day-to-day pill-taking behavior of patients who are taking a medication at least some of the time. In this case, adherence is defined as the percentage of pills a patient takes as prescribed. By convention, a cut-point of $80 \%$ is used to categorize adherence to cardiovascular medications into good and poor adherence groups. A second definition of adherence considers the duration of time a patient remains engaged with a prescription regimen, even if intermittently, before discontinuing the medication prematurely and permanently. With this definition, patients are categorized as non-adherent if they discontinue a medication before a certain time period. Primary nonadherence refers to a situation in which a patient "discontinues" a medication before even filling a prescription once. ${ }^{4}$ More detailed definitions of medication adherence depend on the method of adherence measurement and will be described in more detail below.

\section{Gaps in Knowledge}

Although researchers commonly use a cutpoint of $80 \%$ of pills taken as prescribed to define poor adherence to cardiovascular medications, the optimal cutpoints for categorizing adherence remain poorly understood. The use of the $80 \%$ cutpoint can be traced back to a small blood pressure treatment trial among 230 steelworkers in which the authors found that diastolic blood pressure declined significantly only when participants took more than $80 \%$ of their pills. ${ }^{5}$ While there is strong reason to suspect that the optimal threshold for adherence may vary between medications as a result of differences in their pharmacokinetic and pharmacodynamic properties, there has been scant research to identify whether other thresholds for categorizing adherence to cardiovascular medications are superior. ${ }^{6}$ It is conceivable that in some clinical settings, such as the use of anti-platelet agents immediately after coronary artery stenting, the optimal threshold for defining adherence may be higher and may even approach $100 \%$. In other settings, such as statin use in patients with low cardiovascular risk, a clinically relevant threshold for defining adherence may be considerably lower. Increasing our understanding of the optimal cutpoints for adherence for cardiovascular medications may lead to a more precise understanding of the scope of the adherence problem, the population to which adherence interventions should be targeted, and the scale of resources needed for interventions to improve adherence to specific drug classes.

Another gap in knowledge in defining adherence pertains to the relevance of more precise definitions of medication adherence. With the advent of electronic medication monitors that record the date and time medications are ingested, we can now measure not only the total number of pills a patient took over a time period, but the extent to which the correct number of pills were taken each day (referred to as dosing adherence) and the extent to which these pills were taken during the correct time intervals (referred to as timing adherence). It remains to be determined how much these more precise definitions of medication adherence are relevant to understanding the impact of adherence on cardiovascular outcomes 
To date, most definitions of day-to-day adherence presume that adherence is a stable patient characteristic. Yet, there is evidence that medication adherence may be more accurately understood as a dynamic process. For example, for some patients who have suffered an acute coronary syndrome (ACS), the ACS may serve as a teachable moment that leads to improvements in medication adherence over time. In other post-ACS patients, the stress of the ACS may lead to a worsening of adherence. Statistical approaches such as latent class analysis and adaptive statistical methods can be used to determine whether distinct longitudinal trajectories of adherence (e.g., consistent good adherence versus delayed nonadherence) are present in a population. ${ }^{7,8}$ Future studies should assess whether definitions of adherence that incorporate time-varying patterns can assist efforts to target patient groups with the most worrisome adherence patterns and guide the most appropriate time points for personalized interventions.

\section{Prevalence and Impact of Poor Adherence to Cardiovascular Medications}

\section{Prevalence}

Notwithstanding variability in definitions of adherence, how common is poor adherence to cardiovascular medications? In a meta-analysis of 20 observational studies involving 376,162 patients, a summary estimate of the prevalence of poor adherence across multiple drug classes as measured by pharmacy refill data was $43 \% .{ }^{1}$ This is similar to the estimated prevalence of poor adherence to both cardiovascular and non-cardiovascular medications as reported by the World Health Organization. ${ }^{9}$ These percentages represent summary estimates, and there are substantial differences in adherences to specific cardiovascular medications, even among those prescribed for the same cardiovascular condition. In the case of antihypertensives, adherence ranges from a low of $28 \%$ for $\beta$-blockers to a high of $65 \%$ for angiotensin receptor blockers. ${ }^{10}$ Adherence also varies depending on whether medications are prescribed for primary or secondary prevention with primary prevention typically associated with lower adherence. ${ }^{1}$ Yet, even in the immediate aftermath of acute cardiac events, adherence is suboptimal. In a population cohort of 4,591 post-myocardial infarction (MI) patients, $18 \%$ did not fill their cardiac medications even once in the 4 months after discharge from hospital, ${ }^{11}$ and in a separate cohort of 22,379 post-ACS patients, $60 \%$ discontinued their statin medication within 2 years of hospitalization. ${ }^{12}$ The transition from hospitalization to discharge is a period of particularly high risk for medication errors and non-adherence. ${ }^{13}$ Even in clinical trial settings where patients are carefully selected, substantial rates of poor adherence have been documented. ${ }^{6}$ Hence, irrespective of differences in how and when adherence is measured, poor adherence to cardiovascular medications is highly prevalent across patient populations and cardiovascular drug classes.

\section{Impact}

Poor medication adherence has been associated with a number of adverse health impacts. To begin with, poor adherence is associated with poor control of risk factors such as hypertension and cholesterol. ${ }^{14}$ Unfortunately, clinicians are often unaware that poor adherence underlies the uncontrolled risk factor. This often leads to unnecessary treatment intensification and the potential for adverse effects of overtreatment when patients suddenly take their complete regimen. ${ }^{14}$ Poor adherence is also associated with worse health outcomes. Rasmussen and colleagues showed that survivors of acute MIs who had poor and intermediate adherence to statins were at $25 \%$ and $12 \%$ increased risk of mortality, respectively, compared to survivors with high adherence. ${ }^{15}$ Similarly, Spertus and colleagues showed that patients who prematurely discontinued thienopyridines within 30 days of drug-eluting stent placement were at 9 times increased risk of mortality in the subsequent year, ${ }^{16}$ and our study demonstrated that patients who had poor adherence to 
aspirin (took $<80 \%$ doses correctly) in the month after ACS had nearly twice the risk of morality or recurrent cardiovascular events in the following year. ${ }^{17}$ Poor adherence to heart failure medications has also been associated with increased mortality and heart failure hospitalizations. ${ }^{18}$ Thus, patients with poor medication adherence have strong and consistent associations with worse health outcomes across a range of evidence-based medications and cardiovascular conditions.

Poor adherence also contributes to health care costs. Although poor adherence leads to lower medication costs, it can increase non-medication health care costs by causing suboptimal disease control and increased health care utilization. Using administrative claims data, Sokol and colleagues estimated the health care costs associated with poor adherence to hypertension and hypercholesterolemia medications. ${ }^{19}$ They found that savings from lower medication costs were offset by increased total medical costs driven in part by decreased hospitalizations such that poor adherence was associated with higher overall health care costs. These findings have subsequently been verified in other samples using updated methodologies. ${ }^{20}$ This raises the tantalizing possibility that programs to increase medication adherence may actually provide an opportunity in which investment in health services can both improve health outcomes and lower costs.

\section{Gaps in Knowledge}

Some have questioned the extent to which poor medication adherence directly causes worse health outcomes or whether the association between the two is spurious. They speculate that medication adherence is likely a marker of other favorable health behaviors (e.g., adherence to medical advice in general or to behaviors like exercise and smoking cessation) or socioeconomic characteristics (e.g., access to health care or social support), such that the strong associations between medication adherence and outcomes are primarily due to a "healthy adherer" effect rather than to specific benefits of adhering to a given medication. Evidence in support of the healthy adherer effect comes from several post-hoc analyses of randomized controlled trials in which patients who had better adherence to placebo had better health outcomes than patients who were less adherent to placebo or to a non-harmful treatment. For example, in the in the Beta Blocker Heart Attack Trial, patients who were more adherent to placebo had $62 \%$ lower odds of mortality than patients who were nonadherent to placebo within a year of follow-up. ${ }^{21}$ If this hypothesis is correct, then interventions that are directed toward increasing adherence to specific medications may not have the desired effect on health outcomes.

Another possible explanation for the health benefits of medication adherence is that the act of taking pills may activate a placebo effect. The placebo effect can be defined as a psychophysiologic effect that is derived from expecting a benefit from treatment. ${ }^{22}$ Laboratory studies have shown that the receipt of placebo medications can result in demonstrable biological effects. Moreover, in studies of the impact of adherence to placebo on health outcomes, adjusting for adherence to other health behaviors like smoking and exercise do not reliably weaken the strength of the benefit of adherence to placebo. ${ }^{23}$ These findings challenge the hypothesis that the benefits of adherence to placebo are due to a healthy adherer effect and increase the likelihood that increasing adherence can amplify the biological benefits of the placebo effect.

Additional evidence contrary to the "healthy adherer" hypothesis comes from the retrospective study by Rasmussen and colleagues. ${ }^{15}$ They showed that while poor adherence to statins and renin-angiotensin system inhibitors after MI was associated with increased adverse events, poor adherence to calcium-channel blockers - a drug class not expected to have an impact on post-MI prognosis - was not associated with worse outcomes. ${ }^{15}$ This led the authors to conclude that adherence to specific medications can have specific effects on 
health outcomes. Future studies examining the association between adherence and health outcomes should seek to determine whether the strong association between medication adherence and outcomes is mainly due to drug effect or whether alternative mechanisms such as healthy adherer or placebo effects play a major role. This can be accomplished by carefully measuring potential confounders of the relationship between adherence and outcomes such as health behavior, socioeconomic status, or susceptibility for the placebo effect.

\section{Approaches to Measuring Medication Adherence}

\section{Clinical Judgment}

Clinical judgment represents the most common way medication adherence is assessed by physicians and allied health professionals. Unfortunately, multiple studies have demonstrated that clinicians are poor at judging the extent to which their patients are taking their pills. ${ }^{24}$ Overall, clinicians tend to underestimate the extent of non-adherence in their practice, likely due to a combination of reasons such as lacking awareness of the high prevalence of non-adherence, disbelieving that their own patients would not follow their recommendations, and relying on patient self-reports even though patients often over-report how well they adhere.

\section{Validated Self-Report Tools}

To overcome the inadequacy of relying on clinical judgment, a number of self-report instruments have been developed to measure medication adherence (Table 1). Some selfreport instruments ask about aspects of non-adherent behavior (e.g., unintentionally forgetting medications when travelling, intentionally skipping doses when feeling well) over different time periods. ${ }^{25}$ Others ask patients to recall the extent to which pills were taken during specific time periods using a visual analog scale or single-item questions or combine items asking about the frequency of adherence with items asking about specific adherence behaviors. The test properties of these instruments are often validated against objective measures of adherence or against measures of disease control. ${ }^{26}$ An advantage of these instruments is that they are generally brief, inexpensive, provide immediate feedback to the clinician or researcher, and the responses may help identify some correctable misperceptions about adherence to medications. However, despite their relative simplicity, these scales are at best moderately related to objective measures of adherence and are limited by social desirability and other recall biases. These biases lead the instruments to overestimate how well patients adhere by approximately $10-20 \%$ as compared to objective measures. ${ }^{27}$

Despite their limitations, clinicians can learn some important lessons from validated selfreport instruments. First, in an attempt to decrease over-reporting, many of these tools are prefaced by a script that seeks to normalize non-adherent behavior. Clinicians might apply this strategy when asking whether their own patients have missed doses. In addition, counterintuitively, studies suggest that questionnaires that ask about adherence over a medium to long-term time frame (e.g., two-weeks or longer) are more accurate than those that ask about the most recent period $(<1$ week $) .{ }^{28}$ Hence, to get a more accurate estimate, clinicians should ask about non-adherence more generally rather than focusing on adherence on the day or two preceding the clinic visit. Finally, clinicians should be mindful that selfreports likely represent over-reports of true adherence and hence patients who admit to even some non-adherence behavior should be carefully assessed for potential adherence problems. 


\section{Objective Measures}

Given the high potential for recall bias with self-report measures of adherence, a number of objective measures of medication adherence have been developed. Direct measures of medication intake via drug levels (e.g., digoxin) are not available for most cardiovascular medications. Even when available, they are highly dependent on the pharmacologic properties of the medication and represent imprecise measures of the intensity or duration of pill-taking. Hence, clinicians and researchers must rely on indirect measures of objective medication adherence.

Indirect measures of medication adherence include physiologic or biologic markers, pill counts, pharmacy refill data, and electronic measurement (Table 1). The advantages and disadvantages of these approaches are described below. Physiologic or biologic markers of adherence to cardiovascular medications include blood pressure in the case of antihypertensives, LDL levels in the case of statins, and platelet function tests in the case of aspirin and other anti-platelet agents. Similar to relying on drug levels, while potentially helpful for identifying patients who are non-adherent, such markers depend on the pharmacology of the medication and the biology of the patient. In many cases, these tests cannot distinguish whether a patient is resistant to the drug for biological reasons or whether the patient is truly non-adherent.

Pill counts are often used in research settings, but are often impractical for clinical settings especially if clinicians are expected to count pills for multiple medications. The common patient practice of stockpiling medications at home challenges the accuracy of pill counts. Further, patients may discard pills prior to visits at which they expect pills to be counted which may lead to overestimates of adherence. Pill counts also do not provide information as to whether pills were taken at the time intervals prescribed.

Pharmacy refill data have become increasingly accessible from pharmacy benefit managers such as Medicare Part D and from health systems with closed pharmacy systems such as the Veterans Administration. Refill data can be used to calculate metrics such as the proportion of days covered by medication (PDC, defined as the number of pills dispensed between the first and last refill divided by the number of days between refills) or the time to discontinuation of a medication (defined as the length of time prior to a large gap in medication use). ${ }^{3}$ One important advantage of refill data is that they can be obtained without patients knowing they are being observed. While pharmacy benefit plans have begun sharing these data with clinicians in some settings, they are still not readily available for most clinical encounters and are almost never provided at the point-of-care.

Electronic adherence monitoring is widely regarded as the gold standard for measuring medication adherence in research settings. The most common type of monitors are comprised of special pill caps embedded with an electronic chip that records the date and time when the cap is twisted open. Other versions include pillboxes that record the date and time when each bin is opened and closed. A recently approved system, known as Proteus, records the date and time when a pill enters the stomach. In this system, pills are compounded with a $1 \mathrm{~mm}$ silicon-based sensor that releases a signal to a patch worn on the patient's arm when the sensor comes into contact with stomach acids. The advantage of electronic monitoring is that it allows for tracking not just the number of pills taken, but the intervals between doses and hence can measure whether pills are taken on a day-to-day basis. Electronic monitoring still has limitations. Foremost, it requires adherence to the electronic monitoring system which can disrupt the usual manner in which patients take their medications. For example, a patient who takes their complex medication regimen from a non-electronic pillbox may feel hassled or confused by having to concurrently use an electronic pill bottle to track adherence to one of their cardiovascular medications. In 
response, patients may either decline to use the electronic system or may take some pills in an unmonitored fashion such that the number of pill container openings will not reflect the number of pills ingested. Another limitation of electronic monitors is that, in contrast with pharmacy refill data, they serve as reminders that adherence is being observed which may in turn influence adherence behaviors. Finally, electronic monitors are not yet widely linked with clinical care pathways or covered by health insurance plans such that it is difficult for clinicians to use them to track adherence outside of research settings.

\section{Gaps in Knowledge}

Significant questions remain regarding the optimal tool for measuring medication adherence. In the case of self-report instruments, few data exist pertaining to the head-to-head validity of one instrument versus another. Self-report tools that have been validated in one population (e.g., general medicine patients) may not be appropriate for another (e.g., elderly patients with cognitive deficits). Further, while some studies suggest that asking about a longer time period leads to more accurate measurements, the optimal recall period is not clear. The lack of a high-quality objective gold-standard measure of adherence has complicated the identification of an ideal self-report measure. Nevertheless, given their ease of use, an imperfect measure may be better than no measure and future studies are warranted to assess whether systematically integrating these instruments into clinical pathways as a means of screening for non-adherence has any beneficial effect on health outcomes.

With respect to pharmacy refill data, organizations such as the International Society of Pharmacoeconomics and Outcomes Research have sought to develop standard definitions of adherence $^{3}$, yet, the optimal pharmacy refill measure of adherence remains elusive and a worthy topic for future research. ${ }^{29}$ The increasing availability of automatic refills via mailorder pharmacies threatens the validity of pharmacy refill data as the passive receipt of refills may less clearly reflect pill-taking behavior. The increasing number of low-cost generic medications being filled from large pharmacy chains such as Walmart and Target without claims being sent to pharmacy benefit plans is also making it more difficult to obtain complete refill data. ${ }^{30}$ For now, combining refill data with complimentary measures of adherence (e.g., day-to-day electronic adherence or self-reported adherence) may best capture the full extent to which patients are adherent. Pharmacy refill data will help confirm that patients remain engaged in at least some amount of prescribed medication use, and selfreports or electronic monitoring can measure how well patients are executing regimens on a day-to-day basis.

A major opportunity relevant to the measurement of medication adherence has been technological advances in electronic medication monitors. Until recently, patients had to return electronic monitors to researchers or clinicians in order for the adherence information to be uploaded from the device. Yet, the newest versions of these devices can automatically transmit adherence data via telephone lines or wirelessly using cellular technologies such that medication adherence can be measured remotely and unobtrusively by clinicians or family members. One can imagine a scenario in which electronically measured adherence becomes a standard piece of data that is integrated into the medical record, monitored outside of the confines of the in-person clinic visit, and available in real-time. ${ }^{31}$ If a patient's adherence drops below a certain level, it could trigger a phone call from a care manager or health coach who could seek to problem-solve with a patient how to address the drop in adherence. In the meantime, significant gaps in knowledge remain regarding the acceptability of these devices to patients, the feasibility of integrating their use into the clinical work-flow, and their cost-effectiveness if adopted for wide-spread use. 


\section{Reasons for Poor Medication Adherence}

A growing body of research has sought to understand reasons for non-adherence to cardiovascular medications. ${ }^{29}$ One approach to grouping reasons is to place them into patient, provider, health system, and environmental categories (Figure 1). Patient-level reasons include characteristics such as lack of social support, concerns about medications, and psychological, cognitive, and medical vulnerabilities. Studies that have directly surveyed patients to understand reasons for poor medication adherence have shown that among the patient-level factors, psychological disorders like depression and post-traumatic stress disorder are consistently among the most strongly associated. ${ }^{32}$ Provider-level reasons include factors such as the manner in which clinicians communicate with their patients and the extent to which clinicians favor simpler dosing schedules. ${ }^{33}$ Lack of patient-centered communication, in particular, has been associated with worse adherence. Health system reasons include aspects such as the cost of drug co-pays and access to follow-up care. Environmental reasons relate to more distal influences than the health system and can range from neighborhood characteristics that disrupt easy access to medication refills to media reports that raise concerns about medications.

Some studies have sought to understand reasons for non-adherence by dividing them into unintentional (e.g., forgetting to take medication) and intentional (e.g., intentionally skipping medications) categories. ${ }^{9}$ Yet, studies have shown that many of the factors that underlie intentional non-adherence also play a role in unintentional non-adherence. For example, patients who have more concerns about medications or perceive them to have low importance are more likely to be both less conscientious (i.e., forgetful) about taking them and to intentionally skip doses at times. In such patients, interventions directed at decreasing unintentional medication non-adherence by focusing on reminders without addressing medication beliefs may not be effective. Hence, researchers and clinicians should be cautious in applying distinctions between intentional and unintentional non-adherence.

Investigators have also conceptualized reasons for poor medication adherence as predictors and barriers. Predictors of adherence encompass patient factors like age, gender, or income level that may help explain subsequent medication adherence. Predictors include factors that can be used to identify patients most in need of adherence interventions, but that cannot necessarily be directly modified. We performed a systematic review of predictors of adherence to statins, and identified a U-shaped association between age and adherence - that is, the oldest ( $\geq 70$ years) and youngest $(<50$ years) patients had lower adherence than the middle-aged (50-69 years). ${ }^{34}$ Similar to the findings of other reviews of predictors of cardiovascular medication adherence, we also found that adherence was significantly lower in women, in those with lower incomes, in those with higher prescription copays, and in cases when statins were prescribed for primary prevention. Yet, despite these significant associations, these predictors explained only a small proportion of the variability in medication adherence. Hence, consistent with the conclusions found in other papers, established predictors of adherence are often insufficient to identify individual patients who are likely to be non-adherent, and they should be used cautiously as a means of targeting high-risk populations. ${ }^{35}$

Another approach to understanding reasons for non-adherence is to focus on barriers. In contrast with predictors of adherence, barriers are restricted to potentially modifiable factors that physicians and/or the health care system can attempt to overcome to reduce medication non-adherence. A well-accepted conceptual model for understanding barriers to medication adherence organizes them into those that stem from interactions between 1) the patient and their health providers, 2) the patient and the health care system, and 3) health providers and the health care system. ${ }^{36} \mathrm{We}$ empirically tested components of this model in a cohort of 
stroke survivors from underserved urban communities in New York City and found that among nine potential barriers, only two were associated with poor medication adherence: 1) high concerns about medications (e.g.: worry, disruption, long-term-effects, dependence) and 2) perceived discrimination by the health system as a result of race, ethnicity, education, or income.

Studies that have used qualitative methods to understand reasons for non-adherence to cardiovascular medication have confirmed the importance of patients' concerns about medications as a key barrier. ${ }^{37,38}$ Qualitative studies have also revealed that patients' beliefs about the etiology and consequences of their cardiovascular illness may diverge substantially from physicians' medical models. Specifically, for many patients, the asymptomatic nature of many cardiovascular diagnoses (e.g., hypertension, hypercholesterolemia) calls into question the need for everyday medications that do not provide any benefit that they can feel.

For the practicing clinician, understanding reasons for non-adherence is a fundamental part of the art of medicine. The list of potential reasons for non-adherence is broad, and many of them may only be uncovered through patient-centered, collaborative communication that takes place over a series of clinical visits. Many patients may be embarrassed to admit that they are having trouble affording medications. Others may not have insight into the manner in which mild cognitive impairments or psychological symptoms are playing a role. To identify reasons for non-adherence, clinicians should seek to engage patients in a nonjudgmental fashion and should keep a broad differential, carefully assessing problems affording medications, medication beliefs, cognitive impairment, and psychological symptoms when reasons are not easily ascertained. Clinicians may also reach out to social supports to both gain a greater understanding of issues relevant to non-adherence and to potentially find resources that can reinforce clinician-directed efforts to reduce nonadherence.

\section{Gaps in Knowledge}

There continues to be interest in increasing the understanding of reasons for poor adherence to as these reasons may provide the basis for novel adherence interventions. Given the complexities that underlie non-adherent behavior, future studies will benefit from considering ecological approaches (e.g., obtaining daily patient diaries) that can account for multiple levels of mechanisms and their potential interactions over time. In some cases, nonadherence may only manifest when there is an interaction between underlying vulnerabilities. For example, in a study of adherence in HIV patients, depression, alone, was not associated with medication adherence overall. However, depression was strongly associated with non-adherence during stressful periods that followed major life events. ${ }^{39}$ These types of studies may benefit from technological advances in measurement tools (e.g., smartphone diaries, electronic medication monitors) as well as expansion and linkage of databases of patient records with pharmacy refill data.

Future studies should also explore novel paradigms that can contribute to our understanding of non-adherence. For instance, given the importance of health beliefs to adherent behavior, a more thorough investigation of how communication of health information affects knowledge transfer and subsequent adherence is warranted. ${ }^{33}$ As media and internet sources of health information continue to proliferate, and with the increasing focus on full disclosure of all possible rare adverse effect of medication treatments, it is important to examine whether information overload and over-emphasis of negative effects of cardiovascular drugs is contributing to non-adherence. ${ }^{40}$ 
While patients' with negative beliefs about medications are at risk for non-adherence, these beliefs still explain a small portion of the variation in adherence between patients. In addition, many patients who believe in the importance of cardiovascular medications are still non-adherent. An established social psychological theory known as terror management theory may provide another new paradigm for understanding medication adherence and the frequent discordance between beliefs about medications and pill-taking behavior. This theory posits that human behavior is strongly motivated by an unconscious fear of mortality. ${ }^{41}$ According to this formulation, it is possible that cardiovascular medications function as unconscious reminders of mortality, and that a large part of medication nonadherence is due to patients' efforts to avoid these reminders even though, consciously, they perceive the medications to be important. Future studies may test this theory by using implicit association tests that assess the extent to which patients have unconscious associations between cardiovascular medications and mortality and then determining whether patients with increased negative associations have lower adherence.

Another emerging field for increasing our understanding of the etiology of poor medication adherence comes from social network theory. ${ }^{42}$ In contrast with conventional studies of determinants of health behavior that emphasize characteristics of individual patients, social network studies emphasize the interconnectedness of multiple individuals and characterize the web of social relations between these individuals. There are several reasons to expect that there will be an association between social network characteristics and medication adherence among the members. First, medication adherence has previously been associated with social support, and social support is one of the mechanisms by which social networks exert their effect. Second, the social network may act as a conduit for ideas about medications. Third, members of one's social network may share risks for medical conditions as a result of shared genetics and environment, and hence, are likely to share ideas about the behaviors necessary to control these conditions. If the social network's concerns embrace the medical issues and medication adherence, then it may be a powerful medium through which to disseminate adherence interventions.

\section{Approaches to Improving Medication Adherence}

In response to the considerable morbidity, mortality, and health care costs associated with poor medication adherence, researchers have tested numerous interventions to improve adherence (Table 2). ${ }^{43}$ The preponderance of these interventions have been directed at overcoming barriers at the patient and provider level. These include enhanced counseling through techniques such as motivational interviewing, reminder telephone calls, decision aids, simplification of drug regimens and the development of combination pills, special medication packaging, increased education about medications and the disease, and enhanced care for depression. In one successful cardiovascular medication adherence intervention that sought to overcome patient-provider barriers, patients were randomized to a pharmacy care program that involved standardized medication education, regular follow-up by pharmacists, and medications dispensed in time-specific blister packs versus usual care. ${ }^{44}$ Following the start of the intervention, adherence increased from $61 \%$ to $97 \%$ and there were concomitant improvements in blood pressure and lipid control.

Several interventions have been directed at patient-health system barriers. Thus far, these have been directed at reducing or eliminating the cost of medications. In one such trial, investigators randomized survivors of MI to receiving secondary prevention medications at no cost versus the usual cost of their drug plan. ${ }^{45}$ They found that over the course of the intervention, adherence was poor in both groups, ranging from $35 \%$ to $49 \%$ in the usual cost group and $41 \%$ to $55 \%$ in the free medication group (only 4-6\% higher). Although the intervention did not have an effect on the primary outcome - first major vascular event or 
revascularization - the rate of total major vascular events or revascularization was slightly reduced and the elimination of copayments did not increase total health care spending. Hence, even though eliminating copayments did not have a dramatic impact on adherence, this trial demonstrated that eliminating out-of-pocket medication costs may be a viable component of future adherence interventions.

Several interventions have been specifically designed to address barriers between providers and health systems. Some examples include providing clinicians with increased time to address non-adherence during clinical visits. Other approaches include providing clinicians with access to adherence data being collected by pharmacy refill databases.

\section{Gaps in Knowledge}

Apart from straight-forward adherence interventions that address out-of-pocket medication costs, successful adherence interventions are complex, costly, and require multiple components. Further, the vast majority produce modest improvements in adherence (adherence only improves $4 \%$ to $11 \%$ ), few are proven to improve health outcomes, and even fewer have been tested for lasting benefits. ${ }^{46}$ Moreover, most interventions are directed at only one health condition, and little is known about the effectiveness of any interventions on improving adherence to medications for multiple concurrent medical conditions. Accordingly, the consensus of systematic reviews is that there is a pressing need for innovative approaches to develop medication adherence interventions. ${ }^{43}$

One approach to developing novel adherence interventions will be to improve upon our ability to accurately measure adherence and to test the benefits of incorporating validated tools such as electronic monitoring into clinical practice. Providing this information at the time of clinical visits will help clinicians more accurately identify non-adherence which is a pre-requisite for deciding whether to focus efforts on titrating medications or counseling about adherence.

Another approach may be to develop new approaches to counseling patients to either prevent or reduce non-adherence. Insights from disciplines like health psychology and cognitive theory can be utilized to identify strategies to modify common misperceptions about risks and benefits of important cardiovascular medications. Psychologists may also help us develop counseling techniques that decouple unconscious associations between medications and mortality. We may learn that counseling strategies that emphasize the adverse consequences of missing doses are counterproductive. Yet another approach may be to develop counseling strategies that help patients form stronger habits for pill-taking behavior such that the behavior becomes more automatic and less susceptible to day-to-day changes in motivations and intentions. ${ }^{47}$

There is strong evidence that reducing the frequency of dosing per day and the total number of pills per day can improve medication adherence. Hence, yet another approach to improving adherence may be to maximally simplify cardiovascular drug regimens by combining medications from three or more drug classes (e.g., aspirin, statin, antihypertensives) into a single daily "polypill". Although one of the rationales for the polypill is to ensure increased prescribing of risk-reducing medications, the other rationale is that the simpler drug regimen is will lead to improved adherence. This hypothesis remains to be formally tested and there is a possibility that the strategy may have the opposite effect as a side-effect from a single component of the polypill may lead to missing doses from the entire regimen. ${ }^{48}$

From a health system perspective, an exciting area is leveraging insights from behavioral economics to inform adherence interventions. ${ }^{49}$ One approach might be to provide financial 
incentives for better adherence behavior. Kimmel and colleagues tested whether a lotterybased incentive intervention could improve adherence to warfarin as a means of improving anticoagulation control. Participants in the intervention were entered into a lottery each day in which they could win from $\$ 3$ to $\$ 100$, but were only eligible to collect their winnings if their adherence was confirmed based on electronic measurement. In this small pilot trial of 100 participants, the financial incentive based intervention did not significantly improve anticoagulation in the overall group, but did result in improved control amongst a subgroup of patients with lower anticoagulation control prior to enrollment suggesting that this approach is still deserving of additional research. ${ }^{50}$

Finally, the social network may provide another powerful tool for adherence interventions. With the newest versions of electronic medication monitors, we can now test the effect of sharing objective adherence information with members of the social network. When low adherence is detected, network members may be activated to increase social support for nonadherent members by, for example, helping patients refill prescriptions or calling with reminders to take pills. The social network may also prevent poor adherence as knowing that one's adherence will be made available to one's close contacts may exert social pressure to maintain good adherence. A potential advantage of this social network approach is that it can operate without the need for additional health care resources as the social network, rather than health care professionals, will be the ones to intervene.

A key to success for future interventions may lie in learning how to target the right intervention at the right patient. Thus far, adherence interventions tested in clinical trials have assigned patients to a variety of treatments regardless of a patient's personal barriers. Yet, non-adherence arises out of a complex set of barriers, and the pattern of barriers differs between patients. For example, high drug costs may be a major barrier in a patient without prescription insurance but not in another with full prescription coverage. Similarly, while helpful in some patients, a chronic disease education intervention may not be effective in patients who already have a good understanding of their disease. In contrast, a targeted, tailored approach to an adherence intervention comprised of first identifying non-adherence using validated methods, then determining a patient's individual-level barriers, then matching intervention components to these barriers may be more effective than providing all patients within a group the same intervention.

\section{Summary and Conclusions}

Medication taking is an incredibly complex health behavior influenced by numerous factors, many of which are still just beginning to be understood. From the clinician's perspective, we have learned that key aspects of optimizing adherence include keeping non-adherence on the agenda, asking about it in as non-judgmental a manner as possible, and remaining mindful that there are few definite predictors of who will be non-adherent so it is best to directly ask patients. When clinicians do identify non-adherence, being mindful of patient-centered communication and exploring patients' concerns about medications may be key factors for improving adherence. In the meantime, clinicians can make efforts to simplify regimens as much as possible, prescribe medications with low out-of-pocket costs, make it easy to obtain refills, encourage adherent habits, enlist social supports when possible, and recommend additional tools such as pill boxes, electronic pill bottles with alarms, or other organizing systems to make it easier for patients to remain adherent to their medications on a day-today basis.

From the point of view of the cardiovascular researcher, there continue to be an abundance of opportunities to contribute to the field of adherence research. Increasing our understanding of optimal cutpoints for defining non-adherence; more precisely determining 
the extent to which medication adherence specifically contributes to adverse health outcomes; improving techniques to measure adherence; applying novel paradigms to understand non-adherence, and developing innovative adherence interventions are all promising areas for future research.

The problem of poor medication adherence is unlikely to go away anytime soon, and if anything is likely to grow larger as the population ages and additional medications are discovered that can alter the course of chronic cardiovascular disease. Given the large scope of the problem, both in terms of adverse health impact and health costs, researchers and clinicians might join forces to raise awareness of this issue for key stakeholders in the health system including government, research funders, health insurers, and the pharmaceutical industry. On a promising note, in 2012, the Centers for Medicare and Medicaid Services added medication adherence (as measured by pharmacy refills) as a quality measure that is tied to payment incentives for Medicare Advantage plans. Although the implications of this policy remain to be tested, such policies may help to align incentives to support investments in adherence interventions in the future.

\section{Acknowledgments}

Dr. Kronish is supported by the National Heart, Lung and Blood Institute (K23 HL098359). Dr. Ye is supported by a National Institutes of Health training grant (T32HL007854-16).

\section{Abbreviations}
ACS acute coronary syndrome
MI myocardial infarction
PDC proportion of days covered

\section{References}

1. Naderi SH, Bestwick JP, Wald DS. Adherence to drugs that prevent cardiovascular disease: metaanalysis on 376,162 patients. Am J Med. 2012; 125:882-7. e1. [PubMed: 22748400]

2. Benner JS, Glynn RJ, Mogun H, Neumann PJ, Weinstein MC, Avorn J. Long-term persistence in use of statin therapy in elderly patients. JAMA. 2002; 288:455-61. [PubMed: 12132975]

3. Cramer JA, Roy A, Burrell A, et al. Medication compliance and persistence: terminology and definitions. Value Health. 2008; 11:44-7. [PubMed: 18237359]

4. Fischer MA, Stedman MR, Lii J, et al. Primary medication non-adherence: analysis of 195,930 electronic prescriptions. J Gen Intern Med. 2010; 25:284-90. [PubMed: 20131023]

5. Sackett DL, Haynes RB, Gibson ES, et al. Randomised clinical trial of strategies for improving medication compliance in primary hypertension. Lancet. 1975; 1:1205-7. [PubMed: 48832]

6. Blaschke TF, Osterberg L, Vrijens B, Urquhart J. Adherence to medications: insights arising from studies on the unreliable link between prescribed and actual drug dosing histories. Annual review of pharmacology and toxicology. 2012; 52:275-301.

7. Modi AC, Rausch JR, Glauser TA. Patterns of nonadherence to antiepileptic drug therapy in children with newly diagnosed epilepsy. JAMA. 2011; 305:1669-76. [PubMed: 21521848]

8. Knafl GJ, Schoenthaler A, Ogedegbe G. Secondary analysis of electronically monitored medication adherence data for a cohort of hypertensive African-Americans. Patient preference and adherence. 2012; 6:207-19. [PubMed: 22536057]

9. Organization WH. Adherence to Long-Term Therapies: Evidence for Action. Geneva: World Health Organization; 2003.

10. Kronish IM, Woodward M, Sergie Z, Ogedegbe G, Falzon L, Mann DM. Meta-analysis: impact of drug class on adherence to antihypertensives. Circulation. 2011; 123:1611-21. [PubMed: 21464050] 
11. Jackevicius CA, Li P, Tu JV. Prevalence, predictors, and outcomes of primary nonadherence after acute myocardial infarction. Circulation. 2008; 117:1028-36. [PubMed: 18299512]

12. Jackevicius CA, Mamdani M, Tu JV. Adherence with statin therapy in elderly patients with and without acute coronary syndromes. JAMA. 2002; 288:462-7. [PubMed: 12132976]

13. Kripalani S, Roumie CL, Dalal AK, et al. Effect of a pharmacist intervention on clinically important medication errors after hospital discharge: a randomized trial. Ann Intern Med. 2012; 157:1-10. [PubMed: 22751755]

14. Whooley MA, de Jonge P, Vittinghoff E, et al. Depressive symptoms, health behaviors, and risk of cardiovascular events in patients with coronary heart disease. JAMA. 2008; 300:2379-88. [PubMed: 19033588]

15. Rasmussen JN, Chong A, Alter DA. Relationship between adherence to evidence-based pharmacotherapy and long-term mortality after acute myocardial infarction. JAMA. 2007; 297:177-86. [PubMed: 17213401]

16. Spertus JA, Kettelkamp R, Vance C, et al. Prevalence, predictors, and outcomes of premature discontinuation of thienopyridine therapy after drug-eluting stent placement: results from the PREMIER registry. Circulation. 2006; 113:2803-9. [PubMed: 16769908]

17. Rieckmann N, Burg MM, Kronish IM, Chaplin WF, Schwartz JE, Davidson KW. Aspirin Adherence, Depression and One-Year Prognosis after Acute Coronary Syndrome. Psychother Psychosom. 2011; 80:316-8. [PubMed: 21720196]

18. Gislason GH, Rasmussen JN, Abildstrom SZ, et al. Persistent use of evidence-based pharmacotherapy in heart failure is associated with improved outcomes. Circulation. 2007; 116:737-44. [PubMed: 17646585]

19. Sokol MC, McGuigan KA, Verbrugge RR, Epstein RS. Impact of medication adherence on hospitalization risk and healthcare cost. Med Care. 2005; 43:521-30. [PubMed: 15908846]

20. Roebuck MC, Liberman JN, Gemmill-Toyama M, Brennan TA. Medication adherence leads to lower health care use and costs despite increased drug spending. Health Aff (Millwood). 2011; 30:91-9. [PubMed: 21209444]

21. Horwitz RI, Viscoli CM, Berkman L, et al. Treatment adherence and risk of death after a myocardial infarction. Lancet. 1990; 336:542-5. [PubMed: 1975045]

22. Finniss DG, Kaptchuk TJ, Miller F, Benedetti F. Biological, clinical, and ethical advances of placebo effects. Lancet. 2010; 375:686-95. [PubMed: 20171404]

23. Padula AM, Pressman AR, Vittinghoff E, et al. Placebo adherence and mortality in the Heart and Estrogen/Progestin Replacement Study. Am J Med. 2012; 125:804-10. [PubMed: 22840666]

24. Zeller A, Taegtmeyer A, Martina B, Battegay E, Tschudi P. Physicians' ability to predict patients' adherence to antihypertensive medication in primary care. Hypertens Res. 2008; 31:1765-71. [PubMed: 18971555]

25. Morisky DE, Green LW, Levine DM. Concurrent and predictive validity of a self-reported measure of medication adherence. Med Care. 1986; 24:67-74. [PubMed: 3945130]

26. Shi L, Liu J, Fonseca V, Walker P, Kalsekar A, Pawaskar M. Correlation between adherence rates measured by MEMS and self-reported questionnaires: a meta-analysis. Health Qual Life Outcomes. 2010; 8:99. [PubMed: 20836888]

27. Voils CI, Hoyle RH, Thorpe CT, Maciejewski ML, Yancy WS Jr. Improving the measurement of self-reported medication nonadherence. J Clin Epidemiol. 2011; 64:250-4. [PubMed: 21194887]

28. Lu M, Safren SA, Skolnik PR, et al. Optimal recall period and response task for self-reported HIV medication adherence. AIDS Behav. 2008; 12:86-94. [PubMed: 17577653]

29. Bryson CL, Au DH, Young B, McDonell MB, Fihn SD. A refill adherence algorithm for multiple short intervals to estimate refill compliance (ReComp). Med Care. 2007; 45:497-504. [PubMed: 17515776]

30. Choudhry NK, Shrank WH. Four-dollar generics--increased accessibility, impaired quality assurance. N Engl J Med. 2010; 363:1885-7. [PubMed: 21067379]

31. Asch DA, Muller RW, Volpp KG. Automated hovering in health care--watching over the 5000 hours. N Engl J Med. 2012; 367:1-3. [PubMed: 22716935] 
32. Kronish IM, Edmondson D, Goldfinger JZ, Fei K, Horowitz CR. Posttraumatic stress disorder and adherence to medications in survivors of strokes and transient ischemic attacks. Stroke. 2012; 43:2192-7. [PubMed: 22618380]

33. Ratanawongsa N, Karter AJ, Parker MM, et al. Communication and Medication Refill Adherence: The Diabetes Study of Northern California. Arch Intern Med. 2012:1-9.

34. Mann DM, Woodward M, Muntner P, Falzon L, Kronish I. Predictors of nonadherence to statins: a systematic review and meta-analysis. Ann Pharmacother. 2010; 44:1410-21. [PubMed: 20702755]

35. Steiner JF, Ho PM, Beaty BL, et al. Sociodemographic and clinical characteristics are not clinically useful predictors of refill adherence in patients with hypertension. Circ Cardiovasc Qual Outcomes. 2009; 2:451-7. [PubMed: 20031876]

36. Osterberg L, Blaschke T. Adherence to medication. N Engl J Med. 2005; 353:487-97. [PubMed: 16079372]

37. Kronish IM, Leventhal H, Horowitz CR. Understanding minority patients' beliefs about hypertension to reduce gaps in communication between patients and clinicians. J Clin Hypertens (Greenwich). 2012; 14:38-44. [PubMed: 22235822]

38. Marshall IJ, Wolfe CD, McKevitt C. Lay perspectives on hypertension and drug adherence: systematic review of qualitative research. BMJ. 2012; 345:e3953. [PubMed: 22777025]

39. Bottonari KA, Safren SA, McQuaid JR, Hsiao CB, Roberts JE. A longitudinal investigation of the impact of life stress on HIV treatment adherence. J Behav Med. 2010; 33:486-95. [PubMed: 20577794]

40. Bawden DRL. The dark side of information: overload, anxiety and other paradoxes and pathologies. JIS. 2009; 35:180-91.

41. Goldenberg JL, Arndt J. The implications of death for health: a terror management health model for behavioral health promotion. Psychol Rev. 2008; 115:1032-53. [PubMed: 18954213]

42. Christakis NA, Fowler JH. Social contagion theory: examining dynamic social networks and human behavior. Statistics in medicine. 2012

43. Viswanathan M, Golin CE, Jones CD, et al. Interventions to Improve Adherence to Selfadministered Medications for Chronic Diseases in the United States: A Systematic Review. Ann Intern Med. 2012

44. Lee JK, Grace KA, Taylor AJ. Effect of a pharmacy care program on medication adherence and persistence, blood pressure, and low-density lipoprotein cholesterol: a randomized controlled trial. JAMA. 2006; 296:2563-71. [PubMed: 17101639]

45. Choudhry NK, Avorn J, Glynn RJ, et al. Full coverage for preventive medications after myocardial infarction. N Engl J Med. 2011; 365:2088-97. [PubMed: 22080794]

46. Kripalani S, Yao X, Haynes RB. Interventions to enhance medication adherence in chronic medical conditions: a systematic review. Arch Intern Med. 2007; 167:540-50. [PubMed: 17389285]

47. Gardner B, Lally P, Wardle J. Making health habitual: the psychology of 'habit-formation' and general practice. Br J Gen Pract. 2012; 62:664-6. [PubMed: 23211256]

48. Lafeber M, Spiering W, Singh K, et al. The cardiovascular polypill in high-risk patients. Eur J Prev Cardiol. 2012; 19:1234-42. [PubMed: 22019908]

49. Loewenstein G, Asch DA, Friedman JY, Melichar LA, Volpp KG. Can behavioural economics make us healthier? BMJ. 2012; 344:e3482. [PubMed: 22623635]

50. Kimmel SE, Troxel AB, Loewenstein G, et al. Randomized trial of lottery-based incentives to improve warfarin adherence. Am Heart J. 2012; 164:268-74. [PubMed: 22877814] 


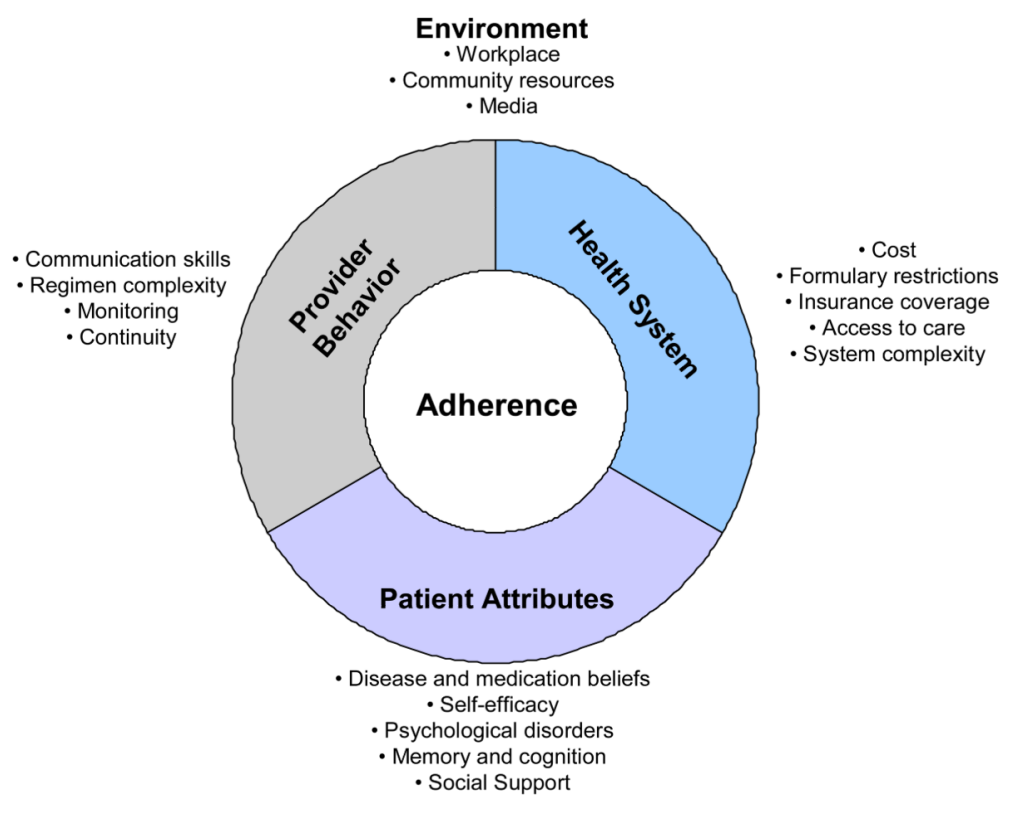

Figure 1.

Reasons for Non-Adherence to Cardiovascular Medications 


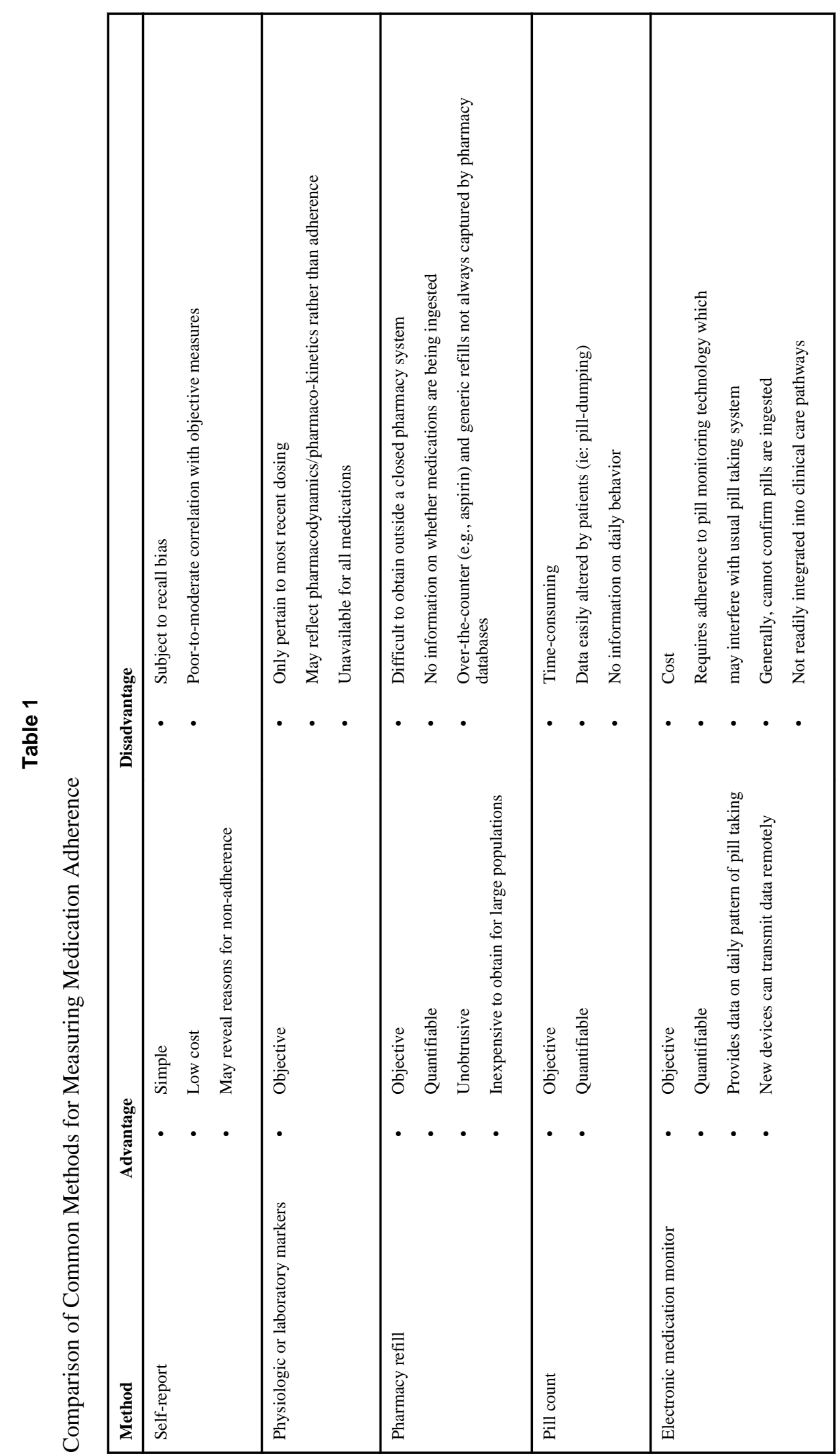

Prog Cardiovasc Dis. Author manuscript; available in PMC 2014 May 01. 


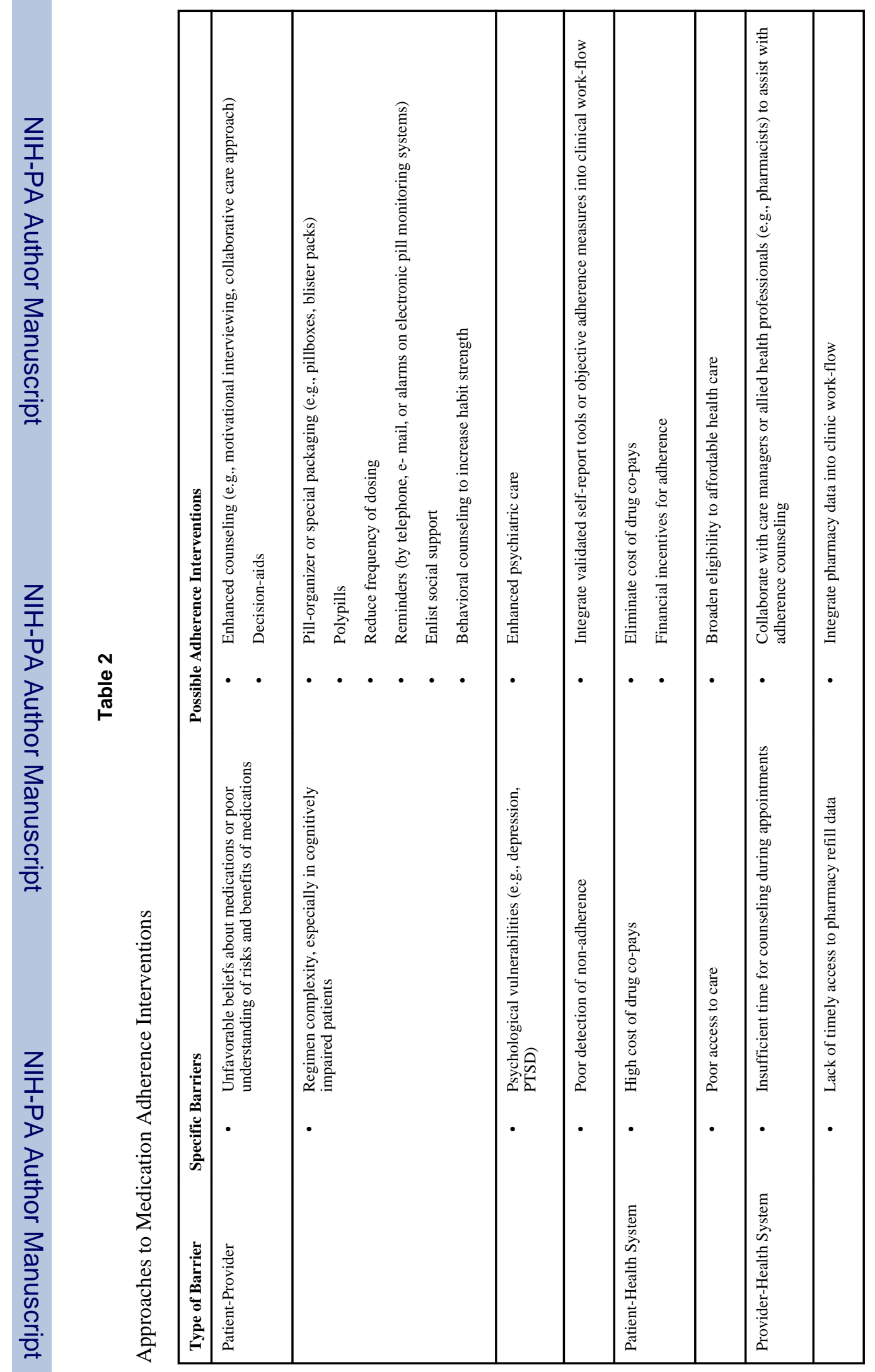

Prog Cardiovasc Dis. Author manuscript; available in PMC 2014 May 01. 